\title{
Fundamental geodesic deformations in spaces of treelike shapes
}

\author{
Aasa Feragen, François Lauze, Mads Nielsen \\ Department of Computer Science, University of Copenhagen \\ \{aasa,francois, madsn\}@diku.dk
}

\begin{abstract}
This paper presents a new geometric framework for analysis of planar treelike shapes for applications such as shape matching, recognition and morphology, using the geometry of the space of treelike shapes. Mathematically, the shape space is given the structure of a stratified set which is a quotient of a normed vector space with a metric inherited from the vector space norm. We give examples of geodesic paths in tree-space corresponding to fundamental deformations of small trees, and discuss how these deformations are key building blocks for understanding deformations between larger trees.
\end{abstract}

\section{Introduction}

Treelike structures form an important class of shapes in image analysis, where they appear for instance in imaging of delivery systems such as airways or vascular systems, or as skeletal structures such as shock graphs describing more general shapes. A natural metric on the set of treelike shapes would have countless applications in pattern recognition, such as shape matching and tree registration $[1,2,4]$, morphometry, recognition and classification. Our goal is to define a metric on the set of trees with geodesics corresponding to natural deformations of the trees, hence reflecting a realistic difference between the shapes.

The problem of studying treelike shapes can be divided into two parts: geometry of the edges, which is related to the shape of planar curves, and topological structure, which describes how the edges are connected. So far, there has been no consistent way to combine the topological and geometric aspects of the treelike shapes into a single metric defined on a space of trees.

In this paper we define tree-space as a geometric object with a given metric, describe its properties and illustrate some geodesic deformations between small trees. These deformations are fundamental in the sense that they represent the sort of local structural changes which we expect to find in geodesics between generic larger trees, and they will form building blocks for recursive algorithms to compute geodesic deformations between trees of any size.

We propose a "Euclidean" metric as defined in (2) below with $p=2$, which simultaneously encodes the topological and geometric differences between the shapes. This approach takes the geometric point of view much further than what has previously been done, and previsouly used metrics can be studied as special cases of our model.

Our metric has fewer geodesics between given points than previously defined metrics on sets of trees, making it suitable for use in statistical analysis. The geodesic paths will pass (generically) through generic (i.e. binary) trees, which is expected of a natural metric. The geodesics are not prone to simplifying the trees, and thus we do not expect means to be topologically simpler than the trees in the datasets. Moreover, the insensitivity of the metric to topological changes which are geometrically "small" makes it less sensitive to noise than other metrics.

The pursuit of such properties is our main motivation for the constructions below.

\section{Background}

Given a treelike structure in an image, we can extract information such as vertices, centerlines and radial information, perhaps in the form of a tubular structure describing how the surface of the tree varies with respect to the centerlines. We arrange this information in the form of an abstract rooted tree describing the topology of the tree, and attach attributes to the edges, where the attributes could be edge length, edge embedding, tubular structure and so on. In this paper we study planar trees whose edges attributes are embeddings of the edges into the plane.

There are previous results concerning metrics on sets of trees: Sebastian et. al. [3] have defined a tree edit distance (TED) type of metric on shock graphs, which adds 


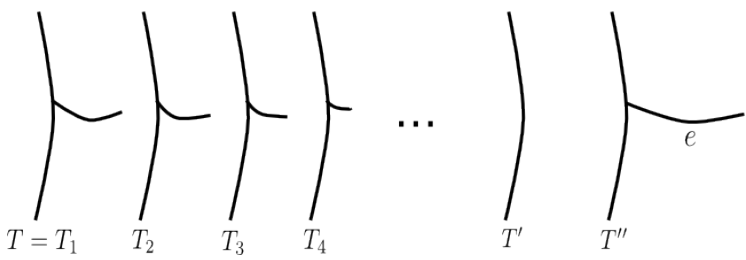

Figure 1. A sequence of trees with a collapsing edge.

the costs of each edge operation needed to deform one tree into another. Wang and Marron [5] have defined a metric on sets of trees which is a combination of a simple TED metric and a Euclidean metric on the space of attributes.

One issue with the TED metric is that it does not distinguish between making edge changes simultaneously or one at a time, and hence we can often find many geodesics of varying complexity between a given pair of trees. A typical choice of geodesic is the one passing through simpler trees (first remove edges, then add), resulting in means that are less complex than the trees being compared.

The metric on tree-space suggested by Wang and Marron [5] is a sum of two metrics; namely a TED metric with cost 1 on each edge addition or removal operation, and a weighted Euclidean metric. One consequence of their definition is that topological changes in the trees are punished much harder than other changes. In particular, a sequence of structurally equivalent trees with a collapsing edge, as in Figure 1, does not converge to the tree with that edge missing; in fact, in Figure 1, with the cost of removing the edge $e$ tuned appropriately, $T^{\prime}$ and $T^{\prime \prime}$ are equally close to being a limit of the sequence. As a result, we expect this metric to be very sensitive to noise in the form of spurious branches due to error in the tree extraction method.

\section{The space of shapes: Embedded trees}

Mathematically, the space of shapes in $\mathbb{R}^{2}$ (planar shapes) is often derived from the space $C^{\infty}\left(M, \mathbb{R}^{2}\right)$ of smooth maps from the set $M$ into $\mathbb{R}^{2}$. Here, each map is a parametrization by the set $M$ of a shape in $\mathbb{R}^{2}$, whose structure mostly resembles that of $M$. If $M$ is a smooth manifold, these mapping spaces are infinitedimensional smooth manifolds. In our case, $M$ will be replaced by a rooted planar tree $T$ (i.e. $T$ encodes combinatorial/topological structure).

We define an embedded tree as a set of maps defined on the edges of a maximal planar rooted binary tree $T$. More precisely, let $T$ denote the standard planar binary tree of depth $m$ (as in Figure 2), and let $E$ denote the



Figure 2. Maximal binary tree of depth $m$.

set of its edges. The set of tree embeddings $f: T \rightarrow \mathbb{R}^{2}$ is defined as a subset of the set

$$
S(T)=\prod_{e \in E}\left\{f_{e} \in C^{\infty}\left([0,1], \mathbb{R}^{2}\right) \mid f_{e}(0)=0\right\},
$$

which is a submanifold of the $\infty$-dimensional manifold $\prod_{e \in E} C^{\infty}\left([0,1], \mathbb{R}^{2}\right)$, since the latter can be identified with the product $\left(\prod_{e \in E} \mathbb{R}^{2}\right) \times S(T)$. Note that using the connectivity information from $T$, we can now build the image of our tree by translating the starting point of each edge to the end point of its parent. That is, the topological structure of the tree corresponding to a point in $S(T)$ is encoded in the tree-space through the topological structure of the underlying tree $T$.

We choose to use a binary maximal tree $T$ because binary trees are generic. Note that we are allowing constant (that is, identically zero) edge maps; this corresponds to collapsing edges as in the middle tree of Figure 3. This allows us to represent non-binary trees and small embedded subtrees of $T$ as maps on the binary tree $T$, making binary trees "sufficiently general".

There are several natural metrics on tree-space induced by norms on $C^{\infty}\left([0,1], \mathbb{R}^{2}\right)$. We discuss briefly two different ways of combining norms on the product (1), defined by $p=1$ or 2 in the following definition: Given a norm $\|\cdot\|$ on $C^{\infty}\left([0,1], \mathbb{R}^{2}\right)$, define the $\|\cdot\|_{p}$ norm of $f: T \rightarrow \mathbb{R}^{2}$ (for $p=1,2, \ldots$ ) as

$$
\|f\|_{p}=\left(\sum_{e \in E}\left\|f_{e}\right\|^{p}\right)^{\frac{1}{p}} .
$$

For $p=1$ this is just the TED metric, when the cost of deforming the edge map $f_{e}$ to the edge map $g_{e}$ is $\left\|f_{e}-g_{e}\right\|$. In this paper, we use the $p=2$ metric, which is similar to the Euclidean metric on finite-dimensional vector spaces, where paths given by simultaneous edge changes are shorter than paths given by changing one edge at a time. As a result, we do not expect the mean shapes to be less complex than the shapes in the original dataset.

\subsection{Finite-dimensional model via landmarks}

We restrict to a finite-dimensional sub-shape-space by representing shapes using a finite number of landmark points. More precisely, we describe the embedded edges using a fixed number $n$ of landmark points 


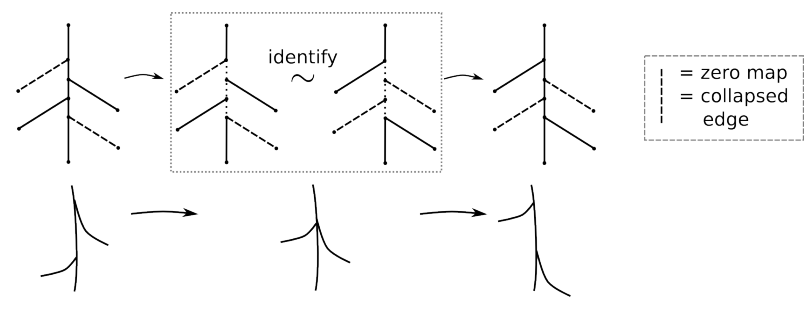

Figure 3. Deformation via identified collapsed trees.

on each edge. Now each annotated shape is represented by a point in $\prod_{e \in E}\left(\mathbb{R}^{2}\right)^{n}=\mathbb{R}^{\sharp E \cdot 2 n}$, which can be naturally and isometrically identified with a subspace of $S(T)$ endowed with the $L^{2}$ norm. The product norm $\|\cdot\|_{2}$ restricts to the Euclidean norm and the induced metric $d(x, y)=\|x-y\|_{2}$ is the Euclidean metric which further emphasizes just how natural the product norm $\|\cdot\|_{2}$ is for the tree-space.

\subsection{Identification of subspaces}

One consequence of the definition of tree-space is that we can obtain the same tree image by collapsing edges in rather (topologically) different trees (such as the two middle top row trees in Figure 3), and thus the same tree image can be represented by several different points in $S(T)$. It seems natural to identify all such trees, but we choose not to, for several reasons.

Firstly, making such identifications corresponds to forcing the tree-space to intersect itself, and as the number of self intersections increases, the geometry of the tree-space becomes more complex - as do the algorithms for computing geodesics.

Secondly, if all topologically similar treeshapes belong to the same tree-subspace (which we would like to be geodesically convex), then we would get some unnatural deformations between disjoint but topologically similar subtrees of larger trees as in Figure 4. Having too many identifications becomes even more of a problem if we study tree-spaces where the attributes are not edge embeddings, but edge length or tubular structure, so that distinct edges can easily have the same attribute.

We therefore restrict ourselves to making identifications that lead to a set of natural geodesics in tree-space which cannot be found in the space $S(T)$. To be more specific, our identifications correspond to the situation of two side branches passing each other along a central "tree trunk" as illustrated in Figure 3, where the top two middle trees are identified. The identification defines an equivalence $\sim$ on $S(T)$ and (by restriction) on $\mathbb{R}^{\sharp E \cdot 2 n}$.

These deformations are fundamental in the sense that together with Euclidean geodesics, which deform edges

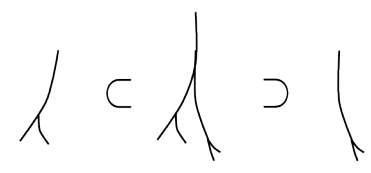

Figure 4. Topologically similar subtrees.

without changing the tree topology, they describe - locally - generic geodesics in tree-space, and we plan to build recursive (with respect to tree size) algorithms computing geodesics between larger trees using the fundamental geodesics as building blocks. See also the snapshots of geodesics presented in Figure 5 and discussed in Section 5.

\section{Geometry of the tree-space}

We define the space of treelike shapes as the quotient of $\mathbb{R}^{\sharp E \cdot 2 n}$ with respect to the equivalence $\sim$. The equivalence induces an identification of linear subspaces of $\mathbb{R}^{\sharp E \cdot 2 n}$, leading to self intersections, and the shape space is not a manifold, but a stratified set, partitioned into strata which are smooth (and, in this case, linear) manifolds. We work in the subspace $X(T)$ of $\mathbb{R}^{\sharp E \cdot 2 n} / \sim$ defined by $P / \sim$, where $P$ is some "orthant" in $\mathbb{R}^{\sharp E \cdot 2 n}$, namely a subset where the variation in each landmark point is spanned by an angle of at most $\pi / 2$. In particular, this means that the angles between different allowed embeddings or landmark point representations of the one same edge are at most $\pi / 2$.

There is a natural distance $\bar{d}$ on $\mathbb{R}^{\sharp E \cdot 2 n} / \sim$ given by

$\bar{d}(\bar{x}, \bar{y})=\inf \left\{\sum_{i=1}^{k} d\left(x_{i}, y_{i}\right) \mid x_{1} \in \bar{x}, y_{i} \sim x_{i+1}, y_{k} \in \bar{y}\right\}$,

where $d$ is the Euclidean metric on $\mathbb{R}^{\sharp E \cdot 2 n}$ and $\bar{x}, \bar{y}$ denote the equivalence classes of $x$ and $y$ in $\prod_{e \in E}\left(\mathbb{R}^{2}\right)^{n} / \sim$. We can prove that

Theorem 3 The distance function $\bar{d}$ restricts to a metric on $X(T)$, and $X(T)$ is a contractible, complete, proper geodesic space.

Due to space considerations, the proof and technical details will appear in a future paper. In plain words, however, this means that given any two points (i.e. embedded trees) in $X(T)$, we can always find a shortest path between them, and the metric $\bar{d}$ is given by the length of the shortest path. In the next section we look at some examples of geodesic deformations.

The reason why we restrict to an orthant is to ensure that the identified subspaces are geodesically convex that is, the geodesic between two points on a subspace does not leave the subspace. In particular, the geodesics 


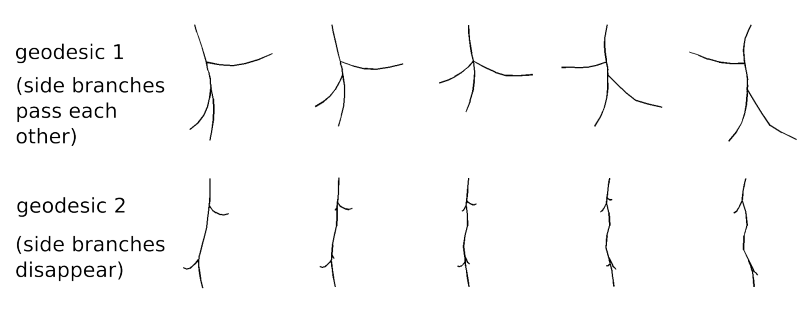

Figure 5. Geodesic paths in tree-space.

in the tree-space are now either straight lines in the Euclidean space, or sequences of straight lines between the endpoint trees and trees in the identified subspaces.

\section{Examples of geodesic paths and distances}

In Figure 5 we see the geodesic paths between two pairs of trees, which are both of the type where one might expect the side branches to pass each other along the trunk in the geodesic deformation. Note that the top geodesic runs through a lower-dimensional stratum corresponding to branches passing, while the bottom geodesic does not go through the lower-dimensional stratum, but corresponds to a single straight line in the tree-space. Movies of the geodesic deformation can be found at http://image.diku.dk/aasa/geodesicmovies/.

This is promising because one would expect (long) branches appearing nearly in the same position to be likely to represent the "same" branch even if the tree topology is different, thus the geodesic between the trees should let the branches pass each other. Meanwhile, (short) branches in arbitrary positions are likely to be noise, and we prefer that such branches shrink and disappear. This tendency is seen even more clearly in Figure 6, where a set of five trees are matched with respect to the distance between them.

\section{Conclusions}

We have defined a space of treelike shapes in $\mathbb{R}^{2}$ and endowed it with a metric which handles branches passing naturally, and whose geodesics do not spend time passing through non-generic trees or trees which are significantly simpler than the endpoint trees. The new metric is less sensitive to noise and false branches than previously defined metrics [5], and we can fit the previously defined metrics on tree-spaces $[3,5]$ into models similar to ours, making comparison easy.

We plan to develop algorithms for computing geodesics between larger trees using the fundamental tree geodesics presented in this paper; to extend the model using additional edge information such as tubular structure; and to develop statistical methods using the new metric in order to work with data consisting of

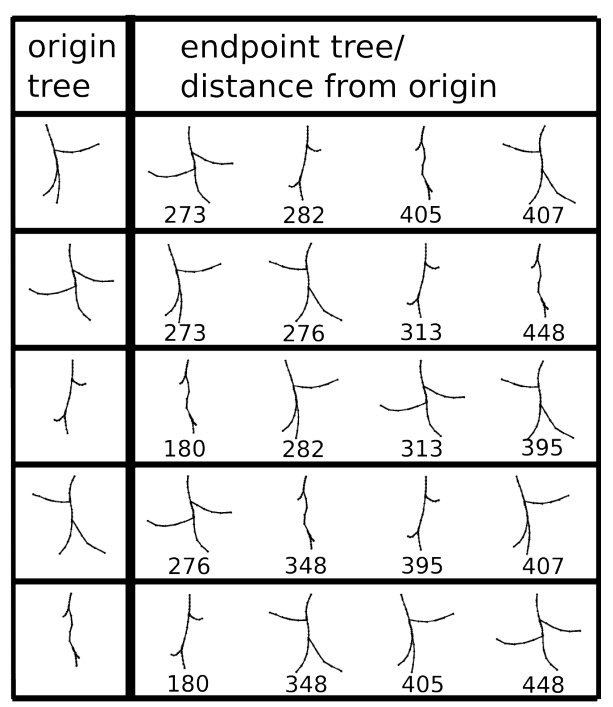

Figure 6. Matching of five small embedded trees using the geodesic distance.

treelike shapes in $\mathbb{R}^{2}$ and $\mathbb{R}^{3}$, for instance with airways or vessels in medical imaging applications.

There are, however, some challenges. We expect calculation of distances between large trees to be computationally expensive (this is also the case with TED metrics). Medical data is typically noisy and we may need to tune our metric or include additional attributes. The extension from trees in $\mathbb{R}^{2}$ to $\mathbb{R}^{3}$ is also not trivial, as we lose the ordered planar structure of the tree.

\section{Acknowledgements}

The authors wish to thank Søren Hauberg and Pechin Lo for helpful discussions.

\section{References}

[1] A. Charnoz, V. Agnus, G. Malandain, L. Soler, and M. Tajine. Tree matching applied to vascular system. GbRPR, 5th IAPR Int. WS. LNCS, 3434:183-192, 2005.

[2] J. H. Metzen, T. Krger, A. Schenk, S. Zidowitz, H.-O. Peitgen, and X. Jiang. Matching of tree structures for registration of medical images. GbRPR, 5th IAPR Int. WS. LNCS, 4538:224-231, 2007.

[3] T. B. Sebastian, P. Klein, and B. Kimia. Recognition of shapes by editing their shock graphs. IEEE Trans. Pattern Anal. Mach. Intell., 26(5):550-571, 2004.

[4] J. Tschirren, G. McLennan, K. Palágyi, E. A. Hoffman, and M. Sonka. Matching and anatomical labeling of human airway tree. IEEE Trans. Medical Imaging, 24(12):1540-1547, 2005.

[5] J. S. Wang, H. and Marron. Object oriented data analysis: Sets of trees. Ann. Statist., 35(5):1849-1873, 2007. 